\title{
Missed Menstrual Period
}

National Cancer Institute

\section{Source}

National Cancer Institute. Missed Menstrual Period. NCI Thesaurus. Code C92832.

The absence of a menstrual period based on the expected start date of the menstrual cycle. 